\title{
Cardiovascular Health: Cross-Generational Considerations
}

\author{
Weedin $\mathrm{E}^{1^{*}}$, Wild $\mathbf{R}^{2}$ and Conant $\mathbf{R}^{1}$
}

${ }^{1}$ Department of Obstetrics and Gynecology, University of Oklahoma Health Sciences Center, Oklahoma City, USA

${ }^{2}$ Department of Obstetrics and Gynecology, Section of Reproductive Endocrinology and Infertility, University of Oklahoma Health Sciences Center, Oklahoma City, USA ${ }^{*}$ Corresponding author: Elizabeth Weedin, Department of Obstetrics and Gynecology, University of Oklahoma Health Sciences Center, Oklahoma City, Oklahoma, USA, Tel: +14052714000; E-mail: Elizabeth-weedin@ouhsc.edu

Received date: August 18, 2015; Accepted date: September 23, 2015; Published date: September 30, 2015

Copyright: @ 2015 Weedin, et al. This is an open-access article distributed under the terms of the Creative Commons Attribution License, which permits unrestricted use, distribution, and reproduction in any medium, provided the original author and source are credited.

\begin{abstract}
Cardiovascular disease (CVD) remains the number one cause of death for women in the United States. Although typically a time of low risk, the reproductive years of a woman's lifetime offer an optimal time to address and prevent future cardiovascular disease by reducing risk factors such as dyslipidemia and early atherosclerosis. Although it is widely known that pregnancy is a state of increased insulin resistance, there are no current reference standards to define normal lipid parameters during pregnancy. Recent animal studies have reported findings that suggest uncontrolled dyslipidemia during pregnancy may potentially predispose or increase the inherent CVD risk for their offspring. Furthermore, women with uncontrolled diabetes, polycystic ovarian syndrome (PCOS) and specific metabolic disorders may all be at yet further increased lifetime risk of dyslipidemia, thereby affecting future health of the mother, her child and future generations. Despite the incomplete understanding of the pathophysiology relating these diseases with potential development of CVD, both in the mother and child, the importance of awareness and improvement in overall health remains paramount.
\end{abstract}

Keywords: Dyslipidemia; Hyperlipidemia; Pregnancy; Cardiovascular disease; Fetal development; Familial hyperlipidemia

\section{Introduction}

Cardiovascular disease (CVD) remains the number one cause of death for women in the United States. Although typically a time of low risk, the reproductive years of a woman's lifetime offers an optimal time to address and prevent future cardiovascular disease by reducing risk factors such as dyslipidemia and early atherosclerosis. Dyslipidemia in pregnancy has historically been considered physiologic with little clinical relevance; recent evidence highlights the importance of preventing or optimizing maternal lipid health before and during pregnancy and the puerperium for the long-term benefit of both mother and the child. Historically it was assumed that maternal cholesterol does not cross the placental barrier; recent evidence suggests otherwise.

Understanding and promoting awareness of opportunities to reduce dyslipidemia before, during, and after pregnancy has major implications for reducing CVD risk for mother, child and for our entire population. Although it is widely known that pregnancy is a state of increased insulin resistance, there are no current reference standards to define normal lipid parameters during pregnancy. This commentary describes the basic understanding of the importance of cholesterol in fetal development as well as maternal management and treatment considerations during pregnancy and beyond.

\section{Cholesterol and Fetal Development}

Cholesterol metabolism is central to normal human development. Sources of fetal cholesterol come from endogenous production through synthesis within the yolk sac as well as from the placenta and from the maternal circulation. Specifically, cholesterol is central to the proper formation of cell membranes, membrane integrity, and the maintenance of cholesterol-rich domains important for membraneassociated signaling cascades. Cholesterol is the precursor to hormones such as sex steroids, vitamin $\mathrm{D}$ and bile acids. In order for exogenous cholesterol to be available for fetal use, the yoke sac and placenta must take up maternal cholesterol via receptor-mediated or receptorindependent transport processes, and thereafter transport the lipids across cellular barriers and/or secrete the maternally derived or newly synthesized cholesterol into fetal circulation $[1,2]$. Human and animal studies have shown that cholesterol may cross the placenta and concentrations are actually higher in the umbilical vein versus the arteries $[2,3]$. The importance of understanding these mechanisms has recently been revealed in small animal studies. In one study in particular, exposure of a fetus to elevated levels of cholesterol and oxidative by-products of cholesterol metabolism has been shown to result in programming of fetal arterial cells with a predisposition to atherosclerosis later in life [4]. Fatty streaks have been found in the aortas of six month-old fetuses of mothers who were hypercholesterolemic [5]. These findings suggest that unbridled dyslipidemia may potentially predispose or increase the inherent CVD risk for their offspring.

\section{Detection, Management and Treatment of Dyslipidemia in Pregnancy}

Pregnancy is a state of insulin resistance and is a cardiometabolic stress test. Lipid and lipoprotein profiles of pregnancy change in parallel. Significant increases in the average circulating values of total cholesterol, low-density lipoprotein cholesterol (LDL-C), and highdensity lipoprotein cholesterol (HDL-C) and triglycerides (TG) are observed during gestation. In the first trimester, there is a noticeable decrease in levels for the first 6 weeks, and then they noticeably increase by the third month or the end of the first trimester. In the third trimester, levels peak near term. Values do not appear however normally to increase above $250 \mathrm{mg} / \mathrm{dL}$ on the average during the entire 
Page 2 of 3

nine months. In contrast, values of triglycerides and total cholesterol often exceed $300 \mathrm{mg} / \mathrm{dL}$ in those with pregnancies that may be complicated by pregnancy-induced hypertension, preeclampsia, gestational diabetes, and/or large for gestational age babies [6]. Understanding this pattern may help clinicians to diagnose dyslipidemia of pregnancy even though reference ranges are not widely standardized.

Women with uncontrolled diabetes, polycystic ovarian syndrome (PCOS) and genetic lipid disorders such as familial hyperlipidemia (FH) may all be at yet further increased risk of dyslipidemia affecting future health of the mother, her child and future generations $[7,8]$. Because $\mathrm{FH}$ and metabolic syndrome are quite common, we believe that the most opportune time to assess lipids is prior to pregnancy or in the early antenatal period soon after a pregnancy is recognized. Screening should then be repeated by or at the 6-week postpartum visit if normal or sooner for management of diagnosed dyslipidemia. Utilizing the puerperal period as a screening opportunity increases compliance and thereby detection rates, especially in the face of our current obesity epidemic. Unfortunately however, complicated or high- risk pregnancies often require multiple providers or a change in a primary obstetrician. As a result of this, lack of continuity can contribute to improper screening, detection and management of dyslipidemia in the long term.

For women who have been diagnosed before pregnancy, any lipidlowering agent may be used for initiation of treatment. If there is a possibility of pregnancy however, or a woman is trying to become pregnant, all medications with the exceptions of bile acid sequestrants and omega 3 fatty acid supplements should be discontinued. It is recommended to stop statin use one to three months before conception, based on expert opinion as the US Food and Drug Administration (FDA) has categorized statins as category $\mathrm{X}$, a medication that has shown previous fetal abnormalities in animals or humans (Tables 1 and 2). These tables are conceptually useful. As of June 30, 2015, the FDA has mandated new labeling changes. These changes should provide better guidance for clinicians as to the risk/ benefit for use in pregnant women, lactating women, and women and men of reproductive potential.

\begin{tabular}{|l|l|}
\hline Pregnancy Category & Description \\
\hline A & $\begin{array}{l}\text { No risk in controlled human studies: Adequate and well-controlled human studies have failed to } \\
\text { demonstrate a risk to the fetus in the first trimester of pregnancy (and there is no evidence of risk in } \\
\text { later trimesters). }\end{array}$ \\
\hline $\mathrm{B}$ & $\begin{array}{l}\text { No risk in other studies: Animal reproduction studies have failed to demonstrate a risk to the fetus } \\
\text { and there are no adequate and well-controlled studies in pregnanat women OR Animal studies have } \\
\text { shown an adverse effect, but adequate and well-controlled studies in pregnant women have failed } \\
\text { to demonstrate a risk to the fetus in any trimester. }\end{array}$ \\
\hline $\mathrm{C}$ & $\begin{array}{l}\text { Risk not ruled out: Animal reproduction studies have shown an adverse effect on the fetus and there } \\
\text { are no adequate and well-controlled studies in humans, but potential benefits may warrant use of } \\
\text { the drug in pregnant women despite potential risks. }\end{array}$ \\
\hline $\mathrm{D}$ & $\begin{array}{l}\text { Positive evidence of risk: There is positive evidence of human fetal risk based on adverse reaction } \\
\text { data from investigational or marketing experience or studies in humans, but potential benefits may } \\
\text { warrant use of the drug in pregnant women despite potential risks. }\end{array}$ \\
\hline $\mathrm{X}$ & $\begin{array}{l}\text { Contraindicated in pregnancy: Studies in animals or humans have demonstrated fetal abnormalities } \\
\text { and/or there is positive evidence of human fetal risk based on adverse reaction data from } \\
\text { investigational or marketing experience, and the risks involved in the use of the drug in pregnant } \\
\text { women clearly outweigh potential benefits. }\end{array}$ \\
\hline $\mathrm{N}$ & \begin{tabular}{l} 
FDA has not yet classified the drug into a specific pregnancy category. \\
\hline
\end{tabular} \\
\hline
\end{tabular}

Table 1: Food and Drug Administration (FDA) pregancy categories.

\begin{tabular}{|l|l|}
\hline Lipid Lowering Agent & Pregnancy Class \\
\hline STATINS & $\mathrm{X}$ \\
\hline FIBRATES & C \\
\hline Ezetimibe & C \\
\hline NIACIN & C \\
\hline Cholestyramine & C \\
\hline Colesevelam & B \\
\hline Mipomersen & B \\
\hline
\end{tabular}

Dyslipidemia during pregnancy should be treated principally by diet and exercise, although it is recommended that it be individualized between each patient and provider. Mediterranean diet and obstetrical recommendations for exercise apply unless there is a need for strict fat free diets because of marked hypertriglyceridemia. Care of pregnancies complicated by other comorbidities such as diabetes types 1 and II, which can be associated with hypertriglyceridemia, should focus first on glycemic control. Medications commonly used for treatment of pregestational or gestational diabetes in pregnancy are glyburide, metformin, and insulin to control blood glucose. A recent randomized trial however has failed to show benefit with Glyburide in gestational diabetic women on pregnancy related outcomes [9]. Attention needs to be focused on residual dyslipidemia in spite of glucose normalization to bring LDL cholesterol and non-HDL cholesterol to normal gestational age adjusted levels. Severe hypertriglyceridemia can be treated with omega 3 fatty acids, parenteral nutrition, plasmapheresis or even gemfibrozil in the mid to late trimesters (class C). With 
Citation: Weedin E, Wild R, Conant R (2015) Cardiovascular Health: Cross-Generational Considerations. Clinics Mother Child Health 12: 190.

Page 3 of 3

medication intervention, lipids should be monitored every six weeks during pregnancy.

\section{Special Considerations}

Patients affected by heritable disorders of lipid metabolism such as familial hyperlipidemia $(\mathrm{FH})$ require special attention, especially in the antenatal period and are best followed in tertiary care centers with experience in this particular patient population. In the pregnant patient with $\mathrm{FH}$, a complete lipid profile assessment is recommended during each trimester. FH can be treated with lifestyle interventions and bile acid sequestrants with monitoring for potential increase in triglyceride levels. If further therapies are required for control, consideration can be given to mipomersen (pregnancy class B) and/or low-density lipoprotein (LDL) apheresis [10].

Another condition that affects a significant proportion of reproductive aged women, approximately $7-22 \%$, is that of polycystic ovary syndrome (PCOS). Women with PCOS are at increased risk for complications of pregnancy, as well as diabetes mellitus, metabolic syndrome, and endometrial cancer [11,12]. Furthermore, irrespective of development of metabolic syndrome, women with PCOS are at greater risk for obstetric complications at baseline, and with obesity being the most common high-risk condition of pregnancy, these women need to be closely monitored throughout gestation. Regarding screening for dyslipidemia in this population, postpartum follow-up is strongly recommended.

\section{Conclusion}

Recent literature indicates that pregnancies affected by hypertensive disorders of pregnancy such as preeclampsia may have endothelial dysfunction that predisposes the mother to increased lifetime CVD risk [13]. This is further compounded by contributions of dyslipidemia, obesity, the presence of the metabolic syndrome or insulin resistance states prior to pregnancy. Despite lack of complete understanding of the pathophysiology relating these diseases with potential development of CVD, both in the mother and child, the importance of awareness and improvement in overall health remains paramount. The understanding of dyslipidemia and hypertensive diseases of pregnancy is constantly evolving. Advancing interest and maximizing research on this topic remains an important scientific, clinical and public health focus.

\section{References}

1. Woollett LA (2001) Fetal lipid metabolism. Front Biosci 6: D536-545.

2. Woollett LA (2005) Maternal cholesterol in fetal development: transport of cholesterol from the maternal to the fetal circulation. Am J Clin Nutr 82: 1155-1161.

3. Yoshida S, Wada Y (2005) Transfer of maternal cholesterol to embryo and fetus in pregnant mice. J Lipid Res 46: 2168-2174.

4. Palinski W, D'Armiento FP, Witztum JL, de Nigris F, Casanada F, et al. (2001) Maternal hypercholesterolemia and treatment during pregnancy influence the long-term progression of atherosclerosis in offspring of rabbits. Circ Res 89: 991-996.

5. Napoli C, D'Armiento FP, Mancini FP, Postiglione A, Witztum JL, et al. (1997) Fatty streak formation occurs in human fetal aortas and is greatly enhanced by maternal hypercholesterolemia. Intimal accumulation of low density lipoprotein and its oxidation precede monocyte recruitment into early atherosclerotic lesions. J Clin Invest 100: 2680-2690.

6. Vrijkotte TG, Krukziener N, Hutten BA, Vollebregt KC, van Eijsden M, et al. (2012) Maternal lipid profile during early pregnancy and pregnancy complications and outcomes: the ABCD study. J Clin Endocrinol Metab 97: 3917-3925.

7. Wild R, Weedin EA, Wilson D (2015) Dyslipidemia in pregnancy. Cardiol Clin 33: 209-215.

8. Wild R, Weedin EA, Gill EA (2015) Women's Health Considerations for Lipid Management. Cardiol Clin 33: 217-231.

9. Casey BM, Duryea EL, Abbassi-Ghanavati M, Tudela CM, Shivvers SA, et al. (2015) Glyburide in Women With Mild Gestational Diabetes: A Randomized Controlled Trial. Obstet Gynecol 126: 303-309.

10. Kusters DM, Homsma SJ, Hutten BA, Twickler MT, Avis HJ, et al. (2010) Dilemmas in treatment of women with familial hypercholesterolaemia during pregnancy. Neth J Med 68: 299-303.

11. Kjerulff LE, Sanchez-Ramos L, Duffy D (2011) Pregnancy outcomes in women with polycystic ovary syndrome: a metaanalysis. Am J Obstet Gynecol 204: 558.

12. Barry JA, Azizia MM, Hardiman PJ (2014) Risk of endometrial, ovarian and breast cancer in women with polycystic ovary syndrome: a systematic review and meta-analysis. Hum Reprod Update 20: 748-758.

13. Charlton F, Tooher J, Rye KA, Hennessy A (2014) Cardiovascular risk, lipids and pregnancy: preeclampsia and the risk of later life cardiovascular disease. Heart Lung Circ 23: 203-212. 